\title{
Nonadiabatic Pauli susceptibility in fullerene compounds
}

\author{
E. Cappelluti ${ }^{1}$, C. Grimaldi ${ }^{2}$ and L. Pietronero ${ }^{1}$ \\ ${ }^{1}$ Dipartimento di Fisica, Universitá di Roma I "La Sapienza", Piazzale A. Moro, 2, 00185 Roma, Italy \\ Istituto Nazionale Fisica della Materia, Unitá di Roma 1, Italy \\ ${ }^{2}$ Département de Microtechnique - IPM, École Polytechnique Fédérale de Lausanne, CH-1015 Lausanne, Switzerland
}

(October 31, 2018)

\begin{abstract}
Pauli paramagnetic susceptibility $\chi$ is unaffected by the electron-phonon interaction in the MigdalEliashberg context. Fullerene compounds however do not fulfill the adiabatic assumption of Migdal's theorem and nonadiabatic effects are expected to be relevant in these materials. In this paper we investigate the Pauli spin susceptibility in nonadiabatic regime by following a conserving approach based on Ward's identity. We find that a sizable renormalization of $\chi$ due to electron-phonon coupling appears when nonadiabatic effects are taken into account. The intrinsic dependence of $\chi$ on the electron-phonon interaction gives rise to a finite and negative isotope effect which could be experimentally detected in fullerides. In addition, we find an enhancement of the spin susceptibility with temperature increasing, in agreement with the temperature dependence of $\chi$ observed in fullerene compounds. The role of electronic correlation is also discussed.
\end{abstract}

PACS number(s): 74.70.Wz, 71.38.-k, 63.20.Kr

\section{INTRODUCTION}

The Pauli susceptibility, $\chi$, of wide-band metals is usually considered as unrenormalized by electron-phonon effects. As pointed out some time ago by Fay and Appel 1 $\chi$ is actually affected by the electron-phonon coupling, the effect having magnitude of order $\lambda \omega_{\mathrm{ph}} / E_{F}$, where $\lambda$ is the electron-phonon coupling, $\omega_{\mathrm{ph}}$ is a typical phonon frequency and $E_{F}$ is the Fermi energy. For wide-band metals, $\omega_{\mathrm{ph}} \ll E_{F}$ and the electron-phonon renormalization of $\chi$ is therefore negligible. This result basically follows from the observation that the lowest order electronphonon correction to $\chi$ is a vertex diagram which, according to Migdal's theorem, 3 is of order $\lambda \omega_{\mathrm{ph}} / E_{F}$. Hence the absence of phonon effects in $\chi$ is just a manifestation of the electron-phonon adiabaticity of normal metals.

This situation may be somewhat different for weak ferromagnetic metals like $\mathrm{ZrZn}_{2}$. electron-phonon corrections compete with the exchange term leading to a possible phonon-dependent ferromagnetic transition temperature. Experiments have however reported only small isotope effects in $\mathrm{ZrZn}_{2}$ with quite large error bars, 1 leaving the problem of phononcorrected weak-ferromagnetism essentially unresolved. The recent discovery of giant isotope shifts in manganites proves however the existence of ferromagnetic materials with imporfant electron-phonon effects in their magnetic properties

The possibility of having electron-phonon dependent spin susceptibility is however not correlated exclusively to the vicinity of magnetic instabilities. In fact as long as $\lambda \omega_{\mathrm{ph}} / E_{F}$ is not negligible, as in very narrow band systems, the electron-phonon vertex contributions are no longer unimportant so that $\chi$ is expected to acquire a phonon renormalization. The most promising candidates for the observation of this effect are the $\mathrm{C}_{60}$-based ma- terials. Fullerene compounds have in fact phonon modes extending up to $0.2 \mathrm{eV}$ and Fermi energies of about 0.3 eV. 5 Therefore, $\omega_{\mathrm{ph}} / E_{F}$ is in principle large suggesting that $\mathrm{C}_{60}$-based metals could be labeled as nonadiabatic systems.

Recently, the finding of a superconducting transition at $T_{c}=52 \mathrm{~K}$ in hole doped $\mathrm{C}_{60}$ single crystals has rised a renewed interest in these materials. 0 Nonzero isotope effects and other properties strongly indicates that superconductixity in fullerides is driven by electron-phonon interaction. 6 However, the description of superconductivity of $\mathrm{Rb}_{3} \mathrm{C}_{60}$ within the traditional Migdal-Eliashberg (ME) theory is found to be inconsistent with respect to the adiabatic hypothesis $\lambda \omega_{\text {prof }} / E_{F} \ll 1$ which is at the basis of the ME theory itself. Instead, by relaxing the adiabatic hypothesis, a generalized formulation which includes nonadiabatic channels in the electron-phonon interaction provides a more self-consistent decription of superconductivity, and suggests that the key ingredient for the high values of $T_{c}$ in $\mathrm{C}_{60}$-based materials is a constructive nonadiabatic interference rather than strong electron-phonon couplings 6.6

In principle, the hypothesis that superconductivity in fullerene compounds is enhanced essentially by nonadiabatic electron-phonon effects can be sustained by the observation of independent signatures of nonadiabaticity. In this respect and according to what we have pointed out at the beginning, Pauli susceptibility is a quantity where such signatures could be found. In this paper, we provide extensive calculations of $\chi$ beyond the adiabatic limit by including nonadiabatic effects at different stages of a perturbation theory in $\lambda \omega_{\mathrm{ph}} / E_{F}$. We find that when $\omega_{\mathrm{ph}} / E_{F}$ is no longer negligible, (i) the paramagnetic spin susceptibility can be considerably reduced with respect to the adiabatic limit, (ii) it acquires a negative isotope effect and (iii) a possible anomalous temperature depen- 
dence at constant sample volume. These features are signatures of nonadiabatic electron-phonon interaction and prediction (ii) and (iii) are susceptible of experimental verification.

\section{PAULI SUSCEPTIBILITY BY WARD'S IDENTITY}

As remarked in the introduction, the effect of electronphonon interaction on spin susceptibility has already attracted some interest in the past mainly in relation to weak antiferromagnets However, different approaches led to different results, 13 reflecting the lacking of a controlled theory.

In our paper we use the functional formalism based on the Baym-Kadanoff technique to derive a conserving derivation of Pauli susceptibility valid for both electronphonon and electron-electron interactions. The Pauli susceptibility $\chi$ is calculated by the knowledge of the spin vertex function which is related to the self-energy via a Ward's identity.

Following the Baym-Kadanoff technique, we introduce an external magnetic field $H$ coupled with the electrons which induces a magnetization $M$. The interaction hamiltonian describing the coupling of $H$ with the electron spins is:

$$
H_{h}=-h \sum_{\mathbf{k}, \sigma} \sigma c_{\mathbf{k} \sigma}^{\dagger} c_{\mathbf{k} \sigma}
$$

where $h=\mu_{\mathrm{B}} H$ and $c_{\mathbf{k} \sigma}^{\dagger}\left(c_{\mathbf{k} \sigma}\right)$ are creation (annihilation) fermionic operators for electrons with wave number $\mathbf{k}$ and spin index $\sigma= \pm 1$. The electron magnetization $M$ due to (11) is given by $M=\mu_{\mathrm{B}} \sum_{\sigma} \sigma n_{\sigma}$ where $n_{\sigma}=\sum_{\mathbf{k}}\left\langle c_{\mathbf{k} \sigma}^{\dagger} c_{\mathbf{k} \sigma}\right\rangle$ and $\langle\cdots\rangle$ denotes the statistical average. We express now the magnetization $M$ in terms of the finite temperature single electron propagator

$$
G_{\sigma}(\mathbf{k}, \tau)=-\left\langle T_{\tau} c_{\mathbf{k} \sigma}(\tau) c_{\mathbf{k} \sigma}^{\dagger}(0)\right\rangle,
$$

where $T_{\tau}$ is the time ordering operator and $\tau$ is the imaginary time. Since $\left\langle c_{\mathbf{k} \sigma}^{\dagger} c_{\mathbf{k} \sigma}\right\rangle=G_{\sigma}\left(\mathbf{k}, 0^{-}\right)$, the magnetization can be expressed as:

$$
\begin{aligned}
M & =\mu_{\mathrm{B}} \sum_{\mathbf{k}, \sigma} \sigma G_{\sigma}\left(\mathbf{k}, 0^{-}\right) \\
& =\mu_{\mathrm{B}} T \sum_{n} \sum_{\mathbf{k}, \sigma} \sigma G_{\sigma}(\mathbf{k}, n) e^{-i \omega_{n} 0^{-}} .
\end{aligned}
$$

In the above expression, $G_{\sigma}(\mathbf{k}, n)$ is the thermal electron propagator which satisfies the following Dyson equation:

$$
G_{\sigma}^{-1}(\mathbf{k}, n)=i \omega_{n}-\epsilon(\mathbf{k})+\mu+h \sigma-\Sigma_{\sigma}(\mathbf{k}, n),
$$

where $\omega_{n}=(2 n+1) \pi T, n=0, \pm 1, \pm 2, \ldots$, are Matsubara frequencies, $\epsilon(\mathbf{k})$ the electron dispersion, $\mu$ the chemical potential and $\Sigma_{\sigma}(\mathbf{k}, n)$ is the electronic self-energy due to the coupling to phonons and to the electron-electron interaction. The spin susceptibility $\chi$ is formally given by $M=\chi H$ where $\chi=[d M / d H]_{0}$ is the derivative of the magnetization at zero field. Hence, from Eq. (3), a general expression for $\chi$ is the following:

$$
\begin{aligned}
\chi(T) & =\mu_{\mathrm{B}}^{2} T \sum_{n} \sum_{\mathbf{k}, \sigma} \sigma\left[\frac{d G_{\sigma}(\mathbf{k}, n)}{d h}\right]_{0} \\
& =-2 \mu_{\mathrm{B}}^{2} T \sum_{n} \sum_{\mathbf{k}} G(\mathbf{k}, n)^{2} \Gamma(\mathbf{k}, n),
\end{aligned}
$$

where $G(\mathbf{k}, n)$ is the electron propagator for zero magnetic field which satisfies the $H \rightarrow 0$ limit of Eq. (4):

$$
G^{-1}(\mathbf{k}, n)=i \omega_{n}-\epsilon(\mathbf{k})+\mu-\Sigma(\mathbf{k}, n) .
$$

In the second term of Eq. (5) we have introduced the spin-vertex function:

$$
\Gamma(\mathbf{k}, n)=\frac{1}{2} \sum_{\sigma} \sigma\left[\frac{d G_{\sigma}^{-1}(\mathbf{k}, n)}{d h}\right]_{0}
$$

In equations (5) and (7), the notation $[\cdots]_{0}$ indicates that the quantity in brackets must be calculated for zero magnetic field. Plugging Eq. (价 into (『) the resulting vertex $\Gamma$ satisfies the Ward's identity:

$$
\Gamma(\mathbf{k}, n)=1-\frac{1}{2} \sum_{\sigma} \sigma\left[\frac{d \Sigma_{\sigma}(\mathbf{k}, n)}{d h}\right]_{0}
$$

and, since $\Sigma_{\sigma}(\mathbf{k}, n)$ is a functional of the electron propagator, a self-consistent relation between (7) and (8) is obtained which permits to calculate the spin susceptibility. At this point, the spin susceptibility $\chi$ can be calculated once the electron self-energy and its magnetic field dependence is known.

\section{NONADIABATIC PAULI SUSCEPTIBILITY}

Electron-phonon interaction is usually neglected in the calculations of the Pauli susceptibility although it can strongly renormalize other physical quantities.10 Indeed it can be shown that the electron-phonon self-energy depends on the external magnetic field $h$ at least as

$$
\lim _{h \rightarrow 0} \Sigma^{\mathrm{ep}}(\mathbf{k}, n) \sim h O\left(\omega_{\mathrm{ph}} / E_{\mathrm{F}}\right),
$$

where $\omega_{\text {ph }}$ characterizes the phonon frequency scale and $E_{\mathrm{F}}$ is the Fermi energy. In wide-band materials the adiabatic ratio $\omega_{\mathrm{ph}} / E_{\mathrm{F}} \ll 1$ and the electron-phonon contribution to $\chi$ can be consequently disregarded. From Eq. (9), we see that electron-phonon effects appear in $\chi$ only at a nonadiabatic level. Hence, any evidence of electronphonon effects on the Pauli susceptibility is thus a direct proof of a nonadiabatic electron-phonon coupling. 


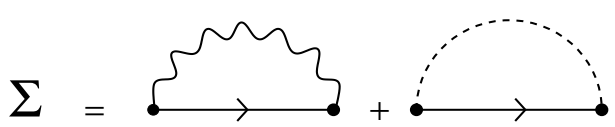

FIG. 1. Electronic self-energy within the non-crossing approximation.

In order to properly include electron-phonon interactions in $\chi$ we need then to explicitely specify the functional form of the nonadiabatic electron-phonon selfenergy. Nonadiabatic effects enter in a twofold way in the electron-phonon self-energy: finite bandwidth effects and vertex diagrams. These two kinds of effects, of course, are of the same order and there is no justification for neglecting vertex corrections with respect to finite bandwidth effects.

In this paper we consider two approximation schemes for the electron-phonon self-energy. The first one is essentially the mean-field theory which corresponds to the non-crossing approximation. It is diagrammatically equivalent to the Migdal-Eliashberg electron-phonon selfenergy without however assuming an infinite electron bandwidth compared to the relevant phonon energies. At this level only finite bandwidth nonadiabatic effects are considered. The second one includes first order electronphonon vertex corrections as well as finite bandwidth effects in the framework on the nonadiabatic Fermi liquid picture 1 Both approximation schemes reduce to the adiabatic Migdal-Eliashberg limit for $\omega_{\mathrm{ph}} / E_{F} \rightarrow 0$.

\section{A. Non-crossing self-energy}

The self-consistent non-crossing approximation neglects the vertex corrections in the electron-phonon selfenergy and for $\omega_{\mathrm{ph}} / E_{F} \ll 1$ reduces to the ME theory of the electron-phonon coupled system. The diagrammatic representation of the electron self-energy is shown in Fig. 11 where the wiggled line represents the phonon propagator and the dashed line the electron-electron Coulomb repulsion. The corresponding compact expression of the non-crossing self-energy reads:

$$
\Sigma_{\sigma}(k)=\sum_{k^{\prime}}\left[V\left(k-k^{\prime}\right)+I e^{-i \omega_{m} 0^{-}}\right] G_{\sigma}\left(k^{\prime}\right),
$$

where $k$ and $k^{\prime}$ are fermionic four-vectors defined as $k \equiv$ $\left(\mathbf{k}, i \omega_{n}\right)$ and $k^{\prime} \equiv\left(\mathbf{k}^{\prime}, i \omega_{m}\right)$. Moreover $\sum_{k} \equiv-T \sum_{n} \sum_{\mathbf{k}}$ and $V\left(k-k^{\prime}\right) \equiv\left|g\left(\mathbf{k}-\mathbf{k}^{\prime}\right)\right|^{2} D\left(k-k^{\prime}\right)$, where $g\left(\mathbf{k}-\mathbf{k}^{\prime}\right)$ is the electron-phonon matrix element and $D\left(k-k^{\prime}\right)$ is the phonon propagator. $I$ is exchange Coulomb interaction which gives rise to the Stoner enhancement factor.

By introducing Eq. (10) into the expression of the spin-vertex function (8) and using Eq. (7) we obtain:

$$
\begin{aligned}
\Gamma(k) & =1-\sum_{k^{\prime}}\left[V\left(k-k^{\prime}\right)+I e^{-i \omega_{m} 0^{-}}\right] \sum_{\sigma} \frac{\sigma}{2}\left[\frac{d G_{\sigma}\left(k^{\prime}\right)}{d h}\right]_{0} \\
& =1+\sum_{k^{\prime}}\left[V\left(k-k^{\prime}\right)+I\right] G\left(k^{\prime}\right)^{2} \Gamma\left(k^{\prime}\right) .
\end{aligned}
$$

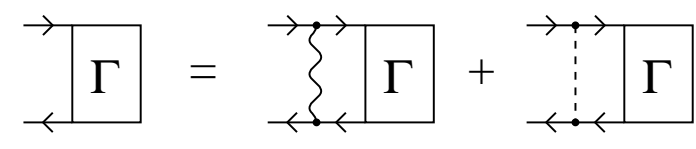

FIG. 2. Diagramatic self-consistent expression of the spin vertex in non-crossing approximation.

The non-crossing approximation for the self-energy leads therefore to a self-consistent ladder equation for $\Gamma(\mathbf{k}, n)$ 1] (Fig. 2). If we consider dispersionless phonons of frequency $\omega_{0}$, the above ladder equations can be rewritten in the extended notation as:

$$
\begin{aligned}
\Gamma(\mathbf{k}, n)= & 1-T \sum_{\mathbf{k}^{\prime}, m}\left[\left|g\left(\mathbf{k}-\mathbf{k}^{\prime}\right)\right|^{2} D(n-m)+I\right] \\
& \times G\left(\mathbf{k}^{\prime}, m\right)^{2} \Gamma\left(\mathbf{k}^{\prime}, m\right),
\end{aligned}
$$

where

$$
D(n-m)=\frac{\omega_{0}^{2}}{\left(i \omega_{m}-i \omega_{m}\right)^{2}-\omega_{0}^{2}} .
$$

The electron propagator appearing in Eq. (11) satisfies the Dyson equation (6) with the zero field limit of the self-energy (10):

$$
\begin{aligned}
\Sigma(\mathbf{k}, n)= & -T \sum_{\mathbf{k}^{\prime}, m}\left|g\left(\mathbf{k}-\mathbf{k}^{\prime}\right)\right|^{2} D(n-m) G\left(\mathbf{k}^{\prime}, m\right) \\
& +I T \sum_{\mathbf{k}^{\prime}, m} G\left(\mathbf{k}^{\prime}, m\right) e^{-i \omega_{m} 0^{-}},
\end{aligned}
$$

In the simplest case of $\mathbf{k}$-independent Coulomb repulsion here considered the second term on the right side of Eq. (14) gives rise just to a constant term which can be absorbed into a redefinition of the chemical potential $\mu^{\prime} \rightarrow \mu$. We can then neglect it since we shall consider only half-filled systems for which we set $\mu^{\prime}=0$.

In an isotropic system the angular dependence of the self-energy and of the vertex function in equations (12) and (14) is negligible and it can be dropped. Therefore, following the same procedure reported in Ref. 11, we replace the electron-phonon interaction $\left|g\left(\mathbf{k}-\mathbf{k}^{\prime}\right)\right|^{2}$ by its average over the Fermi surface:

$$
\left|g\left(\mathbf{k}-\mathbf{k}^{\prime}\right)\right|^{2} \rightarrow\left\langle\left\langle\left|g\left(\mathbf{k}-\mathbf{k}^{\prime}\right)\right|^{2}\right\rangle\right\rangle_{\mathrm{FS}} \equiv g^{2}
$$

where

$$
\left\langle\left\langle\left|g\left(\mathbf{k}-\mathbf{k}^{\prime}\right)\right|^{2}\right\rangle\right\rangle_{\mathrm{FS}}=\frac{\sum_{\mathbf{k}, \mathbf{k}^{\prime}}\left|g\left(\mathbf{k}-\mathbf{k}^{\prime}\right)\right|^{2} \delta[\epsilon(\mathbf{k})] \delta\left[\epsilon\left(\mathbf{k}^{\prime}\right)\right]}{\sum_{\mathbf{k}, \mathbf{k}^{\prime}} \delta[\epsilon(\mathbf{k})] \delta\left[\epsilon\left(\mathbf{k}^{\prime}\right)\right]} .
$$

In this way, the electron self-energy becomes independent of the momentum, $\Sigma(\mathbf{k}, n)=\Sigma(n)$, and the electron propagator becomes at half-filling:

$$
G(\mathbf{k}, n)=\frac{1}{i W_{n}-\epsilon(\mathbf{k})},
$$




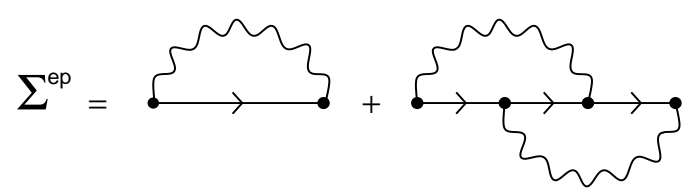

FIG. 3. Vertex corrected electron-phonon self-energy.

where we have set $\Sigma(n)=i \omega_{n}-i W_{n}$. By using a constant density of states, $N_{0}$, the momentum summation in Eq. (14.) is transformed as $\sum_{\mathbf{k}^{\prime}} \rightarrow N_{0} \int_{-E_{F}}^{+E_{F}} d \epsilon$ and, under integration over $\epsilon$, the renormalized frequency $W_{n}$ becomes:

$$
W_{n}=\omega_{n}-\lambda \pi T \sum_{m} D(n-m) \frac{2}{\pi} \arctan \left(\frac{E_{F}}{W_{m}}\right),
$$

where $\lambda=N_{0} g^{2}$ is the electron-phonon coupling constant. By using (15) and (17), also the spin-vertex function (12) becomes momentum independent and, by following the same steps as above, it reduces to:

$$
\Gamma(n)=1+T \sum_{m}[\lambda D(n-m)+I] \frac{2 E_{F}}{W_{m}^{2}+E_{F}^{2}} \Gamma(m) .
$$

Finally, since the self-energy and the spin-vertex function depend only on the frequency, the summation over $\mathbf{k}$ can be readily performed in (5) leading to:

$$
\chi(T)=\chi_{\mathrm{P}} T \sum_{n} \frac{2 E_{F}}{W_{n}^{2}+E_{F}^{2}} \Gamma(n),
$$

where $\chi_{\mathrm{p}}=2 \mu_{\mathrm{B}}^{2} N_{0}$. The spin susceptibility is then obtained by the solution of equations (18), (19), and (20).

\section{B. Vertex corrected self-energy}

In the vertex correction approximation, the electronphonon self-energy $\Sigma^{\mathrm{ep}}$ is modified with respect to the $\mathrm{ME}$ one by the inclusion of the first electron-phonon vertex diagram as shown in Fig. 3. By making use of the condensed notation introduced in Sec. III A, the vertex corrected self-energy can therefore be written as follows:

$$
\begin{aligned}
\Sigma_{\sigma}^{\mathrm{ep}}(k) & =\sum_{k^{\prime}} V\left(k-k^{\prime}\right) G_{\sigma}\left(k^{\prime}\right) \\
& \times\left[1+\sum_{q} V(k-q) G_{\sigma}\left(q-k+k^{\prime}\right) G_{\sigma}(q)\right] .
\end{aligned}
$$

where $q \equiv\left(\mathbf{q}, i \omega_{l}\right)$. The derivative of $\Sigma_{\sigma}^{\mathrm{ep}}(k)$ with respect to $h=\mu_{\mathrm{B}} H$ calculated at zero magnetic field is:

$$
\begin{aligned}
& {\left[\frac{d \sum_{\sigma}^{\mathrm{ep}}(k)}{d h}\right]_{0}=\sum_{k^{\prime}} V\left(k-k^{\prime}\right)\left[\frac{d G_{\sigma}\left(k^{\prime}\right)}{d h}\right]_{0}} \\
& +\sum_{k^{\prime}, q} V\left(k-k^{\prime}\right) V(k-q) G\left(q-k+k^{\prime}\right) G(q)\left[\frac{d G_{\sigma}\left(k^{\prime}\right)}{d h}\right]_{0}
\end{aligned}
$$
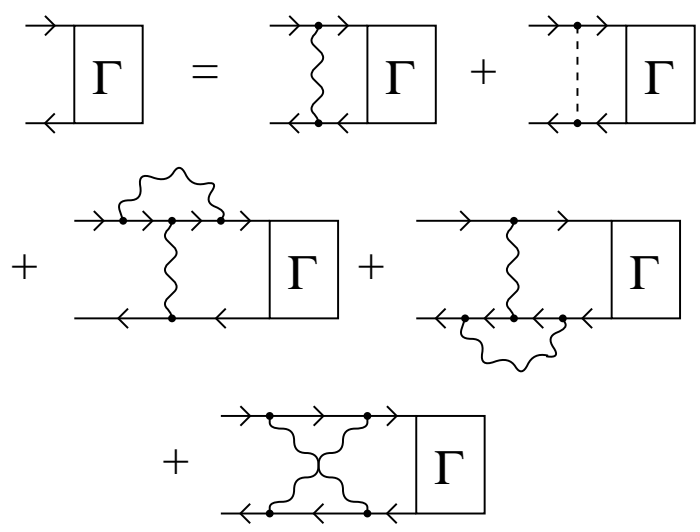

FIG. 4. Diagramatic self-consistent expression of the spin vertex in the vertex corrected theory.

$$
\begin{aligned}
& +\sum_{k^{\prime}, q} V\left(k-k^{\prime}\right) V(k-q) G\left(k^{\prime}\right) G(q)\left[\frac{d G_{\sigma}\left(q-k+k^{\prime}\right)}{d h}\right]_{0} \\
& +\sum_{k^{\prime}, q} V\left(k-k^{\prime}\right) V(k-q) G\left(k^{\prime}\right) G\left(q-k+k^{\prime}\right)\left[\frac{d G_{\sigma}(q)}{d h}\right]_{0}
\end{aligned}
$$

and by re-arranging the 4 -vectors indeces and using (7) and (8) the spin-vertex equation reduces to:

$$
\Gamma(k)=1+\sum_{k^{\prime}}\left[\widetilde{V}\left(k, k^{\prime}\right)+I\right] G\left(k^{\prime}\right)^{2} \Gamma\left(k^{\prime}\right),
$$

where the electron-phonon kernel $\tilde{V}\left(k, k^{\prime}\right)$ is:

$$
\begin{aligned}
\widetilde{V}\left(k, k^{\prime}\right) & =V\left(k-k^{\prime}\right)\left[1+2 \sum_{q} V(k-q) G(q) G\left(q-k+k^{\prime}\right)\right] \\
& +\sum_{q} V(k-q) V\left(q-k^{\prime}\right) G(q) G\left(k+k^{\prime}-q\right) .
\end{aligned}
$$

A graphical representation of (23) and (24) in terms of Feynman diagrams is shown in Fig. 4.

In comparison with Fig. 2, the kernel of the spin susceptibility resulting from the vertex corrected self-energy is modified by additional electron-phonon contributions represented by vertex and cross diagrams. This set of diagrams is therefore quite similar to those encountered in the theory of nonadiabatic superconductivity. They however differ in the orientation of the electron propagators (particle-hole rather than particle-particle contributions) leading to a different momentum dependence of the cross term. The evaluation of the kernel (24) can therefore be carried on by following a scheme similar to the one already employed in previous works. 12

Let us first evaluate the zero-field self-energy entering the electron propagators in Eq. (24). As discussed before, the Coulomb self-energy provides just a constant term which can be absorbed into the definition of the 
chemical potential. The total self-energy reduces therefore to the electron-phonon one which from Eq. (21) can be written in the compact form:

$$
\begin{aligned}
\Sigma(\mathbf{k}, n)= & -T \sum_{m} \sum_{\mathbf{k}^{\prime}}\left|g\left(\mathbf{k}-\mathbf{k}^{\prime}\right)\right|^{2} D(n-m) \\
& \times\left[1+P\left(\mathbf{k}, \mathbf{k}^{\prime} ; n, m\right)\right] G\left(\mathbf{k}^{\prime}, m\right),
\end{aligned}
$$

where we have introduced the vertex function given by:

$$
\begin{aligned}
& P\left(\mathbf{k}, \mathbf{k}^{\prime} ; n, m\right)=-T \sum_{l} \sum_{\mathbf{q}}|g(\mathbf{k}-\mathbf{q})|^{2} D(n-l) \\
& \times G\left(\mathbf{q}-\mathbf{k}+\mathbf{k}^{\prime}, l-n+m\right) G(\mathbf{q}, l) .
\end{aligned}
$$

In similar way as in non-crossing approximation, we eliminate the angular dependence of the self-energy by replacing the whole electron-phonon matrix element, which takes into account the vertex correction, by its average over the Fermi surface:

$$
\begin{aligned}
& \left|g\left(\mathbf{k}-\mathbf{k}^{\prime}\right)\right|^{2}\left[1+P\left(\mathbf{k}, \mathbf{k}^{\prime} ; n, m\right)\right] \rightarrow \\
& \left\langle\left\langle\left|g\left(\mathbf{k}-\mathbf{k}^{\prime}\right)\right|^{2}\left[1+P\left(\mathbf{k}, \mathbf{k}^{\prime} ; n, m\right)\right]\right\rangle\right\rangle_{\mathrm{FS}} \\
& =g^{2}\left[1+\lambda P\left(Q_{c} ; n, m\right)\right]
\end{aligned}
$$

The analytic expression of $P\left(Q_{c} ; n, m\right)$ and its explicit derivation is provided in Appendix A. The parameter $Q_{c}$ is a dimensionless momentum describing the relevant momentum scale of the electron-phonon scattering process. In conventional metals, as the low temperature superconductors, the maximum exchanged phonon momentum $q_{c}$ is about the Debye vector $q_{\mathrm{D}}$ and $Q_{c} \sim q_{\mathrm{D}} / 2 k_{F} \sim 1$. It is well known, however, that in systems with low density of charge carriers, as fullerene's compounds and cuprates, the electrons scatter only with charge fluctuations of long wavelength because of the strong electronic correlation. In fact, in strongly correlated systems, the electrons are surrounded by giant correlation holes which suppress charge density fluctuations with large momenta.13 15 The relevant exchanged phonon scale is thus quite smaller than the Debye vector: $q_{c}<q_{\mathrm{D}}$ and $Q_{c}=q_{c} / 2 k_{F}<1$. As a consequence the effective electron-phonon interaction is suppressed for momenta larger than some cut-off $q_{c}$ which depends on the filling factor and on the Coulomb repulsion. We modelize this situation by introducing a sharp momentum cut-off $q_{c}$. It can be used as a free parameter to distinguish between weak and strong correlation cases, where $q_{c}$ is small for strong correlated systems and of order one for weakly interacting electrons.

As usual, the self-energy effects can be expressed in a convenient form by means of the renormalized frequencies $W_{n}=\omega_{n}\left[1-\Sigma(n) /\left(i \omega_{n}\right)\right]$ obtained by integrating Eq. (25) over the energy. In the vertex corrected theory they fulfil the self-consistent relation:

$$
\begin{aligned}
W_{n}=\omega_{n} & -\pi T_{c} \sum_{m} \lambda\left[1+\lambda P\left(Q_{c} ; n, m\right)\right] D(n-m) \\
& \times \frac{2}{\pi} \arctan \left(\frac{E_{F}}{W_{m}}\right) .
\end{aligned}
$$

By using the momentum independent self-energy (28), the spin-vertex function (23) can be rewritten as:

$\Gamma(\mathbf{k}, n)=1-T \sum_{\mathbf{k}^{\prime}, m}\left[I+\widetilde{V}\left(\mathbf{k}, \mathbf{k}^{\prime} ; n, m\right)\right] \frac{\Gamma\left(\mathbf{k}^{\prime}, m\right)}{\left[i W_{m}-\epsilon\left(\mathbf{k}^{\prime}\right)\right]^{2}}$,

where the nonadiabatic electron-phonon spin kernel $\tilde{V}\left(\mathbf{k}, \mathbf{k}^{\prime} ; n, m\right)$ is given by:

$$
\begin{aligned}
\widetilde{V}\left(\mathbf{k}, \mathbf{k}^{\prime} ; n, m\right) & =\left|g\left(\mathbf{k}-\mathbf{k}^{\prime}\right)\right|^{2} D(n-m) \\
& \times\left[1+2 P\left(\mathbf{k}, \mathbf{k}^{\prime} ; n, m\right)\right]+C\left(\mathbf{k}, \mathbf{k}^{\prime} ; n, m\right) .
\end{aligned}
$$

Here $P\left(\mathbf{k}, \mathbf{k}^{\prime} ; n, m\right)$ is again the first vertex correction given in Eq. (26) and $C\left(\mathbf{k}, \mathbf{k}^{\prime} ; n, m\right)$ is the cross correction whose the explicit expression is given below:

$$
\begin{aligned}
& C\left(\mathbf{k}, \mathbf{k}^{\prime} ; n, m\right)=T \sum_{l} \sum_{\mathbf{q}}|g(\mathbf{k}-\mathbf{q})|^{2}\left|g\left(\mathbf{q}-\mathbf{k}^{\prime}\right)\right|^{2} \\
& \times \frac{D(n-l) D(l-m)}{\left[i W_{l}-\epsilon(\mathbf{q})\right]\left[i W_{n+m-l}-\epsilon\left(\mathbf{k}+\mathbf{k}^{\prime}-\mathbf{q}\right)\right]} .
\end{aligned}
$$

Coherently with the approximations performed on the self-energy and with the Ward's relation Eq. (8), we evaluate $\Gamma(\mathbf{k}, m)$ by replacing the kernel (30) by its momentum average over the Fermi surface:

$$
\widetilde{V}\left(\mathbf{k}, \mathbf{k}^{\prime} ; n, m\right) \rightarrow\left\langle\left\langle\widetilde{V}\left(\mathbf{k}, \mathbf{k}^{\prime} ; n, m\right)\right\rangle\right\rangle_{\mathrm{FS}}=\widetilde{V}\left(Q_{c} ; n, m\right),
$$

where

$$
\begin{aligned}
\widetilde{V}\left(Q_{c} ; n, m\right) & =\lambda D(n-m)\left[1+2 \lambda P\left(Q_{c} ; n, m\right)\right] \\
& +\lambda^{2} C\left(Q_{c} ; n, m\right) .
\end{aligned}
$$

The explicit expression of $C\left(Q_{c} ; n, m\right)$ can be also found in Appendix A.

The final expression of the ladder vertex equation beyond the adiabatic approximation is readily obtained from Eqs. (29)-(33). The result of the integration over the electron energy gives:

$$
\Gamma(n)=1-\lambda T \sum_{m}\left[I+\widetilde{V}\left(Q_{c} ; n, m\right)\right] \frac{2 E_{F}}{W_{m}^{2}+E_{F}^{2}} \Gamma(m) .
$$

Finally, the spin-susceptibility in the vertex corrected approximation is obtained by plugging Eq. (34) into Eq. (20).

\section{RESULTS}

We are now in the position to calculate the Pauli susceptibility $\chi$ and to evaluate the effects on $\chi$ of the 


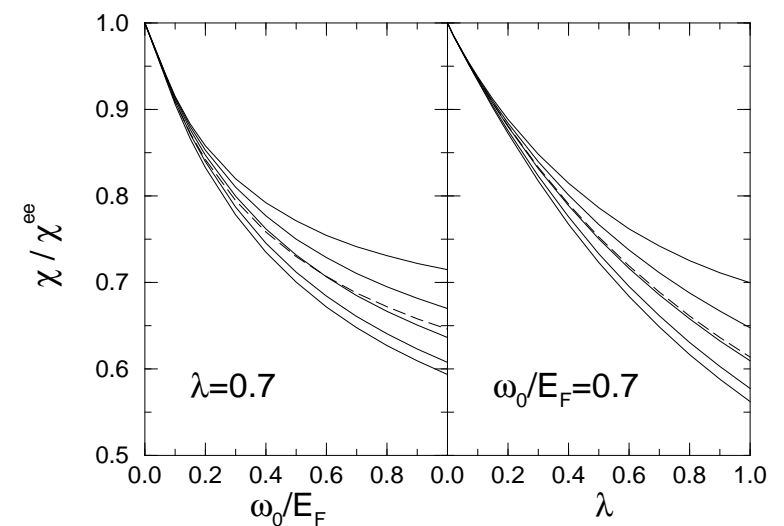

FIG. 5. Spin susceptibility as function of the adiabatic parameter $\omega_{0} / E_{\mathrm{F}}$ and of the electron-phonon coupling $\lambda$ for $N_{0} I=0.4$. Dashed lines represent the spin susceptibility for non-crossing approximation, solid lines for the vertex corrected theory (from the lower to the upper line: $Q_{c}=0.1$, $\left.Q_{c}=0.3, Q_{c}=0.5, Q_{c}=0.7, Q_{c}=1.0\right)$.

electron-phonon interaction, both in the non-crossing approximation and in the vertex corrected theory. Of course, when the adiabatic parameter $\omega_{0} / E_{\mathrm{F}}$ or the electron-phonon coupling constant $\lambda$ are turned to zero $\chi$ would reduce to the simple Stoner enhanced susceptibility:

$$
\lim _{\lambda \rightarrow 0} \chi(T)=\lim _{\omega_{0} / E_{\mathrm{F}} \rightarrow 0} \chi(T)=\frac{\chi_{0}(T)}{1-I N_{0} \frac{\chi_{0}(T)}{\chi_{\mathrm{P}}}},
$$

where $\chi_{0}(T)$ is the free-electron Pauli susceptibility:

$$
\begin{aligned}
\chi_{0}(T) & =-2 \mu_{\mathrm{B}}^{2} T \sum_{n} \sum_{\mathbf{k}} \frac{1}{\left[i \omega_{n}-\epsilon(\mathbf{k})\right]^{2}} \\
& =\chi_{\mathrm{P}}\left[1-2 f\left(E_{F}\right)\right],
\end{aligned}
$$

where $f\left(E_{F}\right)$ is the Fermi distribution function at $E_{F}$.

In the following we will denote the zero electronphonon limit in Eq. (35) as $\chi^{\text {ee }}$ and it would be used as comparison element to evaluate the effects of the electron-phonon interaction.

In Fig. 5 we plot the total spin susceptibility (electronelectron + electron-phonon scattering) as function of the electron-phonon coupling and of the adiabatic parameter for zero temperature. Dashed lines are the results obtained within the non-crossing approximation while the solid lines are the data for the vertex corrected theory. For this latter case we show the results for different values of the momentum cut-off $Q_{c}\left(Q_{c}=0.1,0.3,0.5,0.7\right.$, $1.0)$.

The first main result of Fig. 5 is that the inclusion of the electron-phonon coupling, in the nonadiabatic regime $\omega_{0} / E_{\mathrm{F}} \geq 0$ yields a sensible reduction of $\chi$ with respect to the pure electronic spin susceptibility. As expected this effect vanishes as $\lambda \rightarrow 0$ (right panel) or $\omega_{0} / E_{\mathrm{F}} \rightarrow 0$ (left panel). Note that both the non-crossing and vertex corrected theories yield similar reduction. This is quite

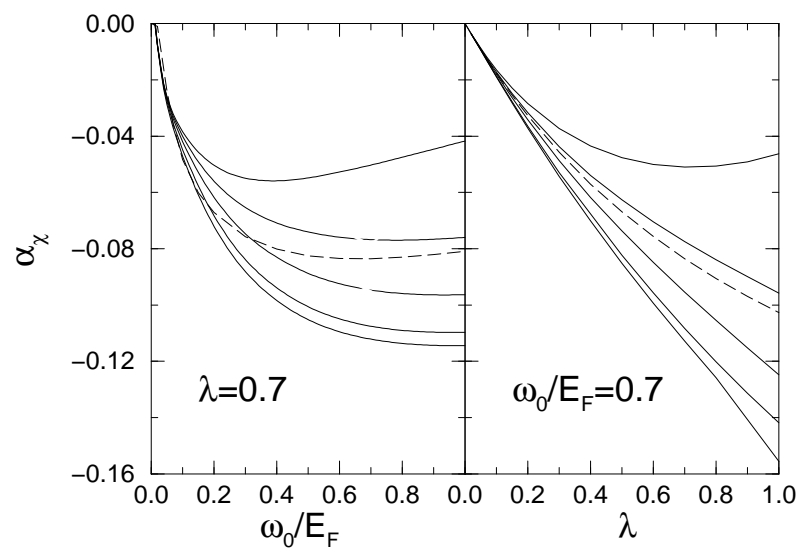

FIG. 6. Isotope effect on the spin susceptibility as function of $\omega_{0} / E_{\mathrm{F}}\left(\lambda=0.7, N_{0} I=0.4\right)$ and as function of $\lambda$ $\left(\omega_{0} / E_{\mathrm{F}}=0.7, N_{0} I=0.4\right)$. Solid lines and dashed line as in previous captions.

different from the situatipnencountered in the superconducting pairing channel, 8,12 where the effect of the vertex corrections is much stronger and highly dependent on $Q_{c}$.

The results of Fig. 5 suggest that some care is needed in estimating the value of the bare density of states from paramagnetic susceptibility measurements. Indeed, our analysis shows that $\chi$ is not simply related to the density of states by Eq. (35). Namely, disregarding the electronphonon effects would lead to a substantial understimation of the bare density of states from a spin susceptibility measurement as long as the electron-phonon interaction is in the nonadiabatic regime.

A more clear signature of the nonadiabatic effects is provided by the isotope dependence of the spin susceptibility. In Fig. 6 we report the numerical calculations of the isotope coefficient $\alpha_{\chi}=-d \log \chi / d \log M$, where $M$ is the ion mass, as a function of the adiabatic ratio $\omega_{0} / E_{F}$ and of $\lambda$. Both the non-crossing (dashed lines) and the vertex corrected (solid lines) theories predict negative values of $\alpha_{\chi}$. Compared to the non-crossing data, the vertex corrected results show for small values of $Q_{c}$ a stronger dependence on $\omega_{0} / E_{F}$ and $\lambda$ leading to $\alpha_{\chi}$ of about -0.1 . The observation of the isotope effect, which should be absent in metals fulfilling the ME framework, could be therefore a stringent evidence of a nonadiabatic electron-phonon coupling. Note that a previous analysis of experimental data of some superconducting properties has demonstrated the failure of the ME theory for $\mathrm{Rb}_{3} \mathrm{C}_{60}$, pointing out the breakdown of Migdal's theorem in fullerides. In this respect, a measurement of $\alpha_{\chi}$ in $\mathrm{C}_{60}$ compounds would represent a direct and independent test of the relevance of nonadiabatic electron-phonon interaction.

From a qualitative point of view, the zero temperature behaviors of both the non-crossing and vertex corrected theories give substantially similar results. A interesting differentiation among the two approximations 


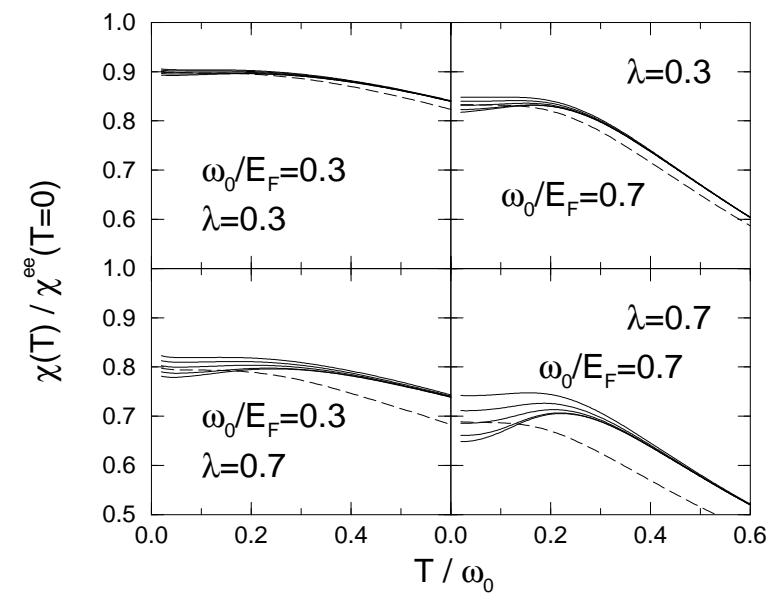

FIG. 7. Temperature dependence of the spin susceptibility for different values of $\omega_{0} / E_{\mathrm{F}}$ and $\lambda$ and $N_{0} I=0.4$. Solid lines and dashed line as in previous captions.

arises in the temperature dependence of $\chi$. In Fig. 10 we report the calculated temperature dependence of the Pauli susceptibility for different values of $\omega_{0} / E_{F}$ and $\lambda$. The non-crossing approximation (dashed lines) yields a monotone decreasing of $\chi$ as the temperature $T$ increases. This is basically due to the phonon cut-off in the ladder equation for the susceptibility, Eq. (19). On the other hand, the vertex corrected $\chi$ (solid lines) has a richer temperature dependence which becomes more important as $\lambda \omega_{0} / E_{F}$ increases. Starting from $T=0$, the basic feature is represented by an initial increase of $\chi$ with $T$ followed by a decreasing for larger temperatures. Although the decreasing part is rather $Q_{c}$ independent, the initial increase of $\chi$ is steeper for lower values of the momentum cutoff. Note for example that for $\lambda=0.7$, $\omega_{0} / E_{F}=0.7$ and $Q_{c}=0.1$, at $T / \omega_{0} \simeq 0.2$ the susceptibility is enhanced by a $8-9 \%$ of its value at $T=0$. Although this increase is rather small, it is nevertheless an interesting feature since it is not related to any increase of the lattice constant due to thermal expansion (the calculations reported here in fact are done for constant volumes). Experimentally in fact, $\chi$ at $T \simeq 300 \mathrm{~K}$ is found to be larger than its value at $T \sim 25 \mathrm{~K}$ of $\sim 39$ $\%$ and $\sim 40 \%$ for $\mathrm{K}_{3} \mathrm{C}_{60}$ and $\mathrm{Rb}_{3} \mathrm{C}_{60}$, respectively $1 \mathrm{1} .17$ Moreover recent data suggest a follewing decrease of $\chi$ in $\mathrm{K}_{3} \mathrm{C}_{60}$ by increasing temperature. 18 The initial increase of $\chi$ with temperature is currently explained by a temperature enhancement of the density of states at the Fermi level due to the thermal expansion of the unit cell. Note however that a power law dependence of the density of state on the lattice constant is not sufficient to reproduce the observed increase of $\chi$, while a stronger dependence like an exponential law needs a quite small value of the Stoner enhancement, $\left(1-N_{0} I\right)^{-1} \simeq 1.3$, to fit the experimental data of $\chi .7$ Such small Stoner enhancement is in contrast to recent Monte Carlo calculations which estimate $\left(1-N_{0} I\right)^{-1} \simeq 319$ Although a detailed study of the effect of the thermal expansion on $\chi$ is beyond the scope of this paper, the results of Fig. 7 suggest that the electron-phonon contribution could be an additional source for the temperature dependence of $\chi$. Of course, a firmer evidence of the role of nonadiabaticity would be the measurement of the temperature dependence of $\chi$ for a constant sample volume, in the spirit therefore of the constant volume resistivity experiment of some years ago.20

An alternative explanation of the non monotone temperature dependence of $\chi$ has recently been attributed to possible effects of mobile ions $\mathrm{K}^{+} 18$ This argument would predict however a finite isotope effect on $\chi$ by alkali isotopic substitution and no carbon isotope effect whereas in nonadiabatic theory an opposite trend is expected.

\section{CONCLUSIONS}

In this paper the nonadiabatic theory of the Pauli spin susceptibility in narrow-band systems has been formulated in order to identify possible signatures of nonadiabatic electron-phonon coupling in fullerides. We have identified peculiar features that can be considered as hallmarks of a relevant electron-phonon coupling in nonadiabatic regime. In particular, an effective reduction of the spin susceptibility by the nonadiabatic electron-phonon coupling has been found in constrast to the conventional ME framework where no electron-phonon renormalization is expected. In addition, we predict a finite negative isotope effect on $\chi$ which we suggest as possible experimental test. The Pauli spin susceptibility of $\chi$ also acquires in nonadiabatic regime an anomalous temperature dependence in qualitative agreement with the experimental data.

The investigation of nonadiabatic effects in $\mathrm{C}_{60}$ compounds attracts new and refreshed interest in the light of the recent experimental indications of an unconventional phonon-based origin of the superconducting pairing. 6 The failure of the conventional theory of superconductivity should not be surprising by considering that one of the basic assumptions of Migdal-Eliashberg theory, namely the adiabatic principle on which Migdal's theorem relies, breaks down in fullerene compounds where phonon frequencies are of the same order of the Fermi energy.

Aimed by these motivations, in the past years we have developed the nonadiabatic theory of superconductivity in narrow band systems that we propose as the theoretical framework to properly describe fullerides and probably cuprates. The inclusion of the nonadiabatic vertex corrections arising from the breakdown of Migdal's theorem has permitted to explain in a natural way some of the experimental features of $\mathrm{C}_{60}$ compounds which appeared anomalous within the ME context. For example, the low density of charge carriers can be regarded as a characteristic element since it is a direct by-product of the band narrowness and as a consequence of the nonadi- 
abatic regime. In addition, the strong electron-electron interaction was shown to yield more attractive pairing channels than repulsive ones in nonadiabatic regime, and to play therefore a positive role with respect to the superconducting onset. More technical anomalies, as the reduction of $T_{c}$ in fullerides by induced disorder, have also received a natural explanation in the context of nonadiabatic theory.

The results of the present work shed new light also on the anomalous $T_{c}$ vs. $\chi$ dependence in ammoniated alkali doped fullerides. In $\left(\mathrm{NH}_{3}\right)_{x} A_{3} \mathrm{C}_{60}$ indeed a sharp reduction of $T_{c}$, together with a weak expansion of the lattice constant $a$, is observed upon increasing of $x .2123$ In addition measurements of Pauli spin susceptibility show that such reduction of $T_{c}$ corresponds to an enhancement of $\chi .223$ This experimental situation looks quite puzzling from the conventional point of view. In ME theory indeed an increase of the lattice constant $a$ is reflected in an enhancement of the bare density of states at the Fermi level $N_{0}$. Both the Pauli susceptibility $\chi \propto N_{0}$ and the critical temperature $\log T_{c} \propto-1 / N_{0}$ are therefore correspondingly expected to increase. Taking into account Stoner enhancement would make even stronger this trend. A theoretical explanation of this situation is still a open issue.

The analysis of the Pauli susceptibility here presented suggests however a new possible interpretation. A crucial point is the observation that the Pauli susceptibility in the nonadiabatic theory, apart from the Stoner factor, does not give a direct probe of the electron density of states but it has to be considered as a quantity renormalized by electron-phonon effects. Even more important is the fact that the renormalization itself depends on electron-phonon properties, so that an increase or decrease of $\chi$ can be tune by electron-phonon properties. Namely, in the model considered in this paper, the Pauli spin susceptibility $\chi$ acquires a significant dependence on $\lambda$ and $Q_{c}$. Figure f shows an enhancement of $\chi$ by decreasing $\lambda$ or $Q_{c}$ (in vertex corrected theory). From an intuitive point of view such an enhancement of $\chi$ should be accompanied by a depletion of $T_{c}$. We quantify this concept by calculating the superconducting critical temperatures in non-crossing approximation and in vertex corrected theory. We consider here $\lambda=0.7$, $Q_{c}=0.1, \omega_{0} / E_{F}=0.7$ and $N_{0} I=0.4$, and we study the $T_{c}$ vs. $\chi$ dependence as function of $\lambda$ (left panel) and $Q_{c}$ (right panel). Numerical calculation are shown in in Fig. 8 for both the theories: solid lines represent the vertex corrected one and the dashed line the non-crossing approximation which does not depend on $Q_{c}$. The results shown in Fig. 8 suggest therefore that the driving element of the anomalous $T_{c}$ vs. $\chi$ behaviour could be the electron-phonon coupling in nonadiabatic regime and the weak lattice expansion of secondary importance. A microscopic relation between ammonia intercalation and possible modifications of the electron-phonon interaction is still missing. The high critical temperature of $\left(\mathrm{NH}_{3}\right)_{4} \mathrm{Na}_{2} \mathrm{CsC}_{60}$ however seems indicate that an impor-

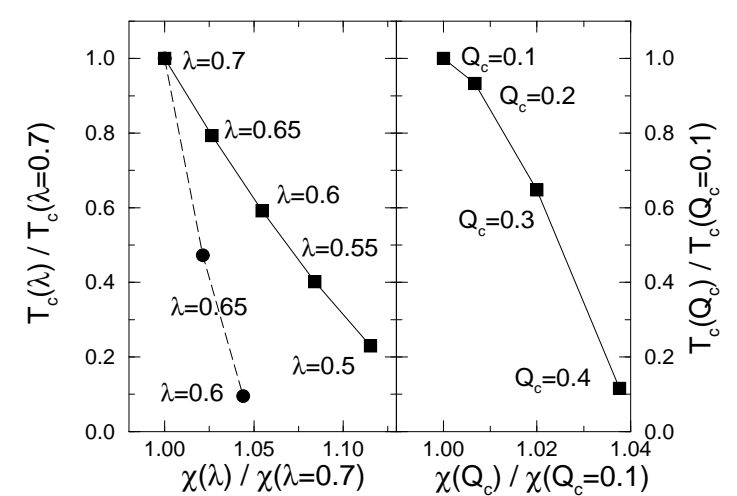

FIG. 8. Critical temperature versus Pauli spin susceptibility in nonadiabatic regime by varying $\lambda$ (left panel) and $Q_{c}$ (right panel).

tant role can be played by the displacement of the alkali cations from the center of the interstitial sites and by the splitting of the crystal field.

\section{APPENDIX A:}

Aim of this appendix is to provide the analytical evaluation of the electron-phonon vertex correction $P\left(\mathbf{k}, \mathbf{k}^{\prime} ; n, m\right)$ and cross function $C\left(\mathbf{k}, \mathbf{k}^{\prime} ; n, m\right)$ and of their average over the momentum transfer. The following derivation is an improvement of the previous calculations presented in the appendix of Ref. 9 by explicitely including the self-consistent self-energy renormalization. In order to do that a different procedure is employed. Let us consider first the vertex correction, Eq. (26), reported below for convenience:

$$
\begin{aligned}
& P\left(\mathbf{k}, \mathbf{k}^{\prime} ; n, m\right)=-T \sum_{l} \sum_{\mathbf{q}}|g(\mathbf{k}-\mathbf{q})|^{2} D(n-l) \\
& \times \frac{1}{i W_{l}-\epsilon(\mathbf{q})} \frac{1}{i W_{l-n+m}-\epsilon\left(\mathbf{q}-\mathbf{k}+\mathbf{k}^{\prime}\right)},
\end{aligned}
$$

We consider a model for the electron-phonon matrix element which simulates the effects of the strong electronic correlation. Strong electron correlation has been shown in literature to favour the forward eleftren-phonon scattering at small transferred momenta. $13-15$ Following Ref. 9, we therefore schematize the electron-phonon matrix $|g(\mathbf{q})|^{2}$ as:

$$
|g(\mathbf{q})|^{2}=\frac{g^{2}}{Q_{c}^{2}} \Theta\left(Q_{c}-Q\right)
$$

where $Q=q / 2 k_{F}, Q_{c}=q_{c} / 2 k_{F}$ and $k_{F}$ is the Fermi wave vector. $\Theta$ is the Heaviside step function and $q_{c}$ is a momentum cut-off which depends on the degree of correlation in the system: $q_{c}$ is smaller for more correlated materials.

The explicit inclusion of the self-energy in $W_{n}$ does not allow for an analytical integration on the Matsubara 
frequencies. In contrast with Ref. 9 we use thus the alternative procedure to evaluate first the integration over the momenta leaving the summation over the frequencies untouched. In evaluating the integration over the Brillouin zone, we employ some basilar approximations which are justified by the dimensionless cut-off $Q_{c}$ of Eq. (A2) supposed to be small (strong correlation case). In this context, we expand the electronic dispersion term $\epsilon\left(\mathbf{p}-\mathbf{k}+\mathbf{k}^{\prime}\right)$ as:

$$
\epsilon\left(\mathbf{p}-\mathbf{k}+\mathbf{k}^{\prime}\right) \simeq \epsilon(\mathbf{p})+2 E_{F} Q \alpha \cos \phi
$$

where $E_{F}=v_{F} k_{F}$ and $\alpha$ and $\phi$ are the polar angles between $\mathbf{q}$ and $\mathbf{k}$. In writing Eq. A3, we have assumed a isotropic system and set $|\mathbf{k}|=\left|\mathbf{k}^{\prime}\right|=|\mathbf{q}|=k_{F}, Q=$ $\left|\mathbf{k}-\mathbf{k}^{\prime}\right| / 2 K_{F}$. The smallness of the second term of Eq. (A3) is enforced by the $\Theta$-functions of $g(\mathbf{q})^{2}$ present in Eq. (A1) and in front of the vertex function [see Eq. (27)].

In similar way, the leading order of the $\Theta$-function appearing in Eq. (A1) reads:

$$
\Theta\left(q_{c}-|\mathbf{k}-\mathbf{q}|\right) \simeq \Theta\left(2 Q_{c}-\alpha\right) \text {. }
$$

Finally, the sum over the Brillouin zone can be expressed in terms of the same angular coordinates $\alpha, \phi$ :

$$
\sum_{\mathbf{p}}=\int_{-\pi}^{\pi} \frac{d \phi}{2 \pi} \int_{0}^{\pi} \frac{d \alpha \sin \alpha}{2} \int_{-E_{F}}^{E_{F}} N(\epsilon) d \epsilon .
$$

By making use of Eqs. (A3), A4), (A5) in Eq. (A1) we obtain the expression for the electron-phonon vertex function:

$$
\begin{aligned}
& P\left(\mathbf{k}, \mathbf{k}^{\prime} ; n, m\right)=\frac{\lambda}{Q_{c}^{2}} T \sum_{l} D(n-l) \int_{-\pi}^{\pi} \frac{d \phi}{2 \pi} \int_{0}^{2 Q_{c}} \frac{d \alpha \alpha}{2} \\
& \times \int_{-E_{F}}^{E_{F}} d \epsilon \frac{1}{i W_{l}-\epsilon} \frac{1}{i W_{l-n+m}-\epsilon-2 E_{F} Q \alpha \cos \phi},
\end{aligned}
$$

where, coherently with the previous model, we have assume a constant $\operatorname{DOS} N(\epsilon)=N_{0}$.

It is convenient rewrite Eq. (A6) in the form:

$$
\begin{aligned}
& P\left(\mathbf{k}, \mathbf{k}^{\prime} ; n, m\right)=\frac{\lambda}{Q_{c}^{2}} T \sum_{l} D(n-l) \int_{-\pi}^{\pi} \frac{d \phi}{2 \pi} \\
& \int_{0}^{2 Q_{c}} \frac{d \alpha \alpha}{2} \frac{2 E_{F} Q \alpha \cos \phi-i\left[W_{l}-W_{l-n+m}\right]}{\left[2 E_{F} Q \alpha \cos \phi\right]^{2}+\left[W_{l}-W_{l-n+m}\right]^{2}} \\
& \int_{-E_{F}}^{E_{F}} d \epsilon\left[\frac{\epsilon+i W_{l}}{\epsilon^{2}+W_{l}^{2}}-\frac{\epsilon+2 E_{F} Q \alpha \cos \phi+i W_{l-n+m}}{\left(\epsilon+2 E_{F} Q \alpha \cos \phi\right)^{2}+W_{l-n+m}^{2}}\right] .
\end{aligned}
$$

A very important role on the structure of the electron-phonon vertex function is played by the term in the second line of Eq. (A7). It contains a factor $1 /\left[\left(2 E_{F} Q \alpha \cos \phi\right)^{2}+\left(W_{l}-W_{l-n+m}\right)^{2}\right]$ which is evidently nonanalitic for $Q \rightarrow 0, W_{l} \rightarrow W_{l-n+m}$. The different behaviour of this term with respect to the opposite regimes $Q /\left[W_{l}-W_{l-n+m}\right] \ll 1$ or $Q /\left[W_{l}-W_{l-n+m}\right] \gg$ 1 make arise the difference between the static and dynamic limits and characterizes the main feature of the momentum-frequency structure of $P$. In contrast to that, the integrand in the third line of Eq. (A7) is basically regular in the whole momentum-frequency space. Hence, in the small $Q$ expansion justified by the correlation cut-off parameter $Q_{c}$, this latter term can be safely expanded at the second order in $Q$ without losing important features of the vertex function.

By integrating on $\epsilon$ and expanding in $Q$ we are then left with:

$$
\begin{aligned}
& P\left(\mathbf{k}, \mathbf{k}^{\prime} ; n, m\right)=-\frac{\lambda}{Q_{c}^{2}} T \sum_{l} D(n-l) \int_{-\pi}^{\pi} \frac{d \phi}{2 \pi} \\
& \int_{0}^{2 Q_{c}} \frac{d \alpha \alpha}{2} \frac{A(n, m, l)-B(n, m, l)\left(2 E_{F} Q \alpha \cos \phi\right)^{2}}{\left[2 E_{F} Q \alpha \cos \phi\right]^{2}+\left[W_{l}-W_{l-n+m}\right]^{2}}
\end{aligned}
$$

where

$$
\begin{aligned}
A(n, m, l)= & \left(W_{l}-W_{l-n+m}\right)\left[\arctan \left(\frac{E_{F}}{W_{l}}\right)\right. \\
& \left.-\arctan \left(\frac{E_{F}}{W_{l-n+m}}\right)\right], \\
B(n, m, l)= & \left(W_{l}-W_{l-n+m}\right) \frac{E_{F} W_{l-n+m}}{\left[E_{F}^{2}+W_{l-n+m}^{2}\right]^{2}} \\
& -\frac{E_{F}}{E_{F}^{2}+W_{l-n+m}^{2}} .
\end{aligned}
$$

The last two integrals on $\alpha$ and $\phi$ can now be performed analytically giving:

$$
\begin{aligned}
& P\left(\mathbf{k}, \mathbf{k}^{\prime} ; n, m\right)=\lambda P\left(Q, Q_{c} ; n, m\right) \\
& =-\lambda T \sum_{l} D(n-l)\{2 B(n, m, l) \\
& +\frac{A(n, m, l)-B(n, m, l)\left[W_{l}-W_{l-n+m}\right]^{2}}{\left(2 E_{F} Q Q_{c}\right)^{2}} \\
& \left.\times\left[\sqrt{1+\left(\frac{2 E_{F} Q Q_{c}}{W_{l}-W_{l-n+m}}\right)^{2}}-1\right]\right\} .
\end{aligned}
$$

The average over the momentum $Q$ is finally obtained from Eq. (27). Also in this case, the integration can be done analytically and the resulting averaged vertex function $P\left(Q_{c} ; n, m\right)$ becomes:

$$
\begin{aligned}
& P\left(Q_{c} ; n, m\right)=T \sum_{l} D(n-l)\{B(n, m, l) \\
& +\frac{A(n, m, l)-B(n, m, l)\left[W_{l}-W_{l-n+m}\right]^{2}}{\left(2 E_{F} Q_{c}^{2}\right)^{2}} \\
& \times\left[\sqrt{1+\left(\frac{4 E_{F} Q_{c}^{2}}{W_{l}-W_{l-n+m}}\right)^{2}}-1\right. \\
& \left.\left.-\ln \left(\frac{1}{2} \sqrt{1+\left(\frac{4 E_{F} Q_{c}^{2}}{W_{l}-W_{l-n+m}}\right)^{2}}\right)\right]\right\} .
\end{aligned}
$$


The calculation of the cross function, Eq. (31), follows basically the same lines reported above. The main difference with respect to the vertex function lies in the momentum dependence, which we handle in the same way as reported in Ref. 8. Therefore, in Eq. (31) we set $g(\mathbf{k}-\mathbf{q})^{2} g\left(\mathbf{q}-\mathbf{k}^{\prime}\right)^{2} \simeq g(\mathbf{k}-\mathbf{q})^{2} g\left(\mathbf{k}-\mathbf{k}^{\prime}\right)^{2}$, which is an approximation valid for small values of $Q_{c}$. Concerning the electronic dispersion, we expand $\epsilon\left(\mathbf{k}+\mathbf{k}^{\prime}-\mathbf{q}\right)$ in the following way:

$$
\begin{aligned}
\epsilon\left(\mathbf{k}+\mathbf{k}^{\prime}-\mathbf{q}\right) \simeq & \epsilon(\mathbf{q})+E_{F}\left(1-Q^{2}\right) \alpha^{2} \\
& -2 E_{F} Q \sqrt{1-Q^{2}} \alpha \cos \phi
\end{aligned}
$$

where the angles $\alpha$ and $\phi$ have the same meaning as before. The integration over the energy and the angles can now be performed and, up to the leading order in the momentum transfer, the cross function becomes independent of $Q=\left|\mathbf{k}-\mathbf{k}^{\prime}\right| / 2 k_{F}$. Hence, for small values of $Q_{c}$, the momentum average leaves the cross function unaltered and, from Eqs. (32,33), we obtain

$$
\begin{aligned}
& C\left(Q_{c} ; n, m\right)=T \sum_{l} D(n-l) D(l-m)\{2 B(n,-m, l) \\
& +\frac{A(n,-m, l)-B(n,-m, l)\left(W_{l}-W_{l-n-m}\right)^{2}}{2 E_{F}^{2} Q_{c}^{2}\left|W_{l}-W_{l-n-m}\right|} \\
& \left.\times \arctan \left(\frac{4 E_{F} Q_{c}^{2}}{\left|W_{l}-W_{l-n-m}\right|}\right)\right\}
\end{aligned}
$$

where the functions $A$ and $B$ are reported in Eqs. (A9, A10).

${ }^{1}$ D. Fay and J. Appel, Phys. Rev. B 20, 3705 (1979); ibid. 22, 1461 (1980).

${ }^{2}$ A. B. Migdal, Zh. Eksp. Teor. Fiz. 34, 1438 (1958) [Sov. Phys. JETP 34, 996 (1958)].

${ }^{3}$ C. P. Enz and B. T. Matthias, Z. Physik B 33, 129 (1979).

${ }^{4}$ G. S. Knapp, E. Corenzwit, and C. W. Chu, Solid State Commun. 8, 639 (1970).

${ }^{5}$ G. M. Zhao, K. Conder, H. Keller, and K. A. Müller, Nature (London) 381, 676 (1996).

${ }^{6}$ O. Gunnarsson, Rev. Mod. Phys. 69, 575 (1997).

${ }^{7}$ J. H. Schön, Ch. Kloc, and B. Batlogg, Nature (London) 408, 549 (2000).

${ }^{8}$ E. Cappelluti, C. Grimaldi, L. Pietronero, and S. Strässler, Phys. Rev. Lett. 85, 4771 (2000).

${ }^{9}$ C. Grimaldi, L. Pietronero, and S. Strässler, Phys. Rev. Lett. 75, 1158 (1995); L. Pietronero, S. Strässler, and C. Grimaldi, Phys. Rev. B 52, 10516 (1995); C. Grimaldi, L. Pietronero, and S. Strässler, Phys. Rev. B 52, 10530 (1995).

${ }^{10}$ G. Grimvall, The Electron-Phonon Interaction in Metals (North-Holland, Amsterdam, 1981).

${ }^{11}$ C. Grimaldi and L. Pietronero, Europhys. Lett. 47, 681 (1999).
${ }^{12}$ M. Scattoni, C. Grimaldi, and L. Pietronero, Europhys. Lett. 47, 588 (1999).

13 M. Grilli and C. Castellani, Phys. Rev. B 50, 16880 (1994).

${ }^{14}$ R. Zeyher, and M. Kulic̀, Phys. Rev. B 53, 2850 (1996).

${ }^{15}$ M. Kulič, Phys. Rep. 338, 1 (2000).

${ }^{16}$ K. Tanigaki, M. Kosaka, T. Manako, Y. Kubo, I. Hirosawa, K. Uchida, and K. Prassides, Chem. Phys. Lett. 240, 627 (1995).

17 J. Robert, P. Petit, T. Yildirim, and J. E. Fischer, Phys. Rev. B 57, 1226 (1998).

${ }^{18}$ N. Čegar, F. Simon, S. Garaj, L. Forró, B. Ruzicka, L. Degiorgi, V. Brouet, and L. Mihály, cond-mat/0002447.

${ }^{19}$ F. Aryasetiawan, O. Gunnarsson, E. Koch, and R. M. Martin, Phys. Rev. B 55, R10165 (1997).

${ }^{20}$ W. A. Vareka and A. Zettl, Phys. Rev. Lett. 72, 4121 (1994).

${ }^{21}$ H. Shimoda, Y. Iwasa, Y. Miyamoto, Y. Maniwa, and T. Mitani, Phys. Rev. B 54, 15653 (1996).

${ }^{22}$ Y. Maniwa, D. Sugiura, K. Kume, K. Kikuchi, Y. Achiba, I. Hirosawa, K. Tanigaki, H. Shimoda, and Y. Iwasa, Phys. Rev. B 54, 6861 (1996).

${ }^{23}$ M. Riccò, T. Shiroka, A. Sartori, F. Bolzoni, and M. Tomaselli, Europhys. Lett. 53, 762 (2001). 